\title{
Extending the Solvent-Free MALDI Sample Preparation Method
}

\author{
Scott D. Hanton and David M. Parees \\ Air Products and Chemicals Inc., Allentown, Pennsylvania, USA
}

\begin{abstract}
Matrix-assisted laser desorption/ionization (MALDI) mass spectrometry is an important technique to characterize many different materials, including synthetic polymers. MALDI mass spectral data can be used to determine the polymer average molecular weights, repeat units, and end groups. One of the key issues in traditional MALDI sample preparation is making good solutions of the analyte and the matrix. Solvent-free sample preparation methods have been developed to address these issues. Previous results of solvent-free or dry prepared samples show some advantages over traditional wet sample preparation methods. Although the results of the published solvent-free sample preparation methods produced excellent mass spectra, we found the method to be very time-consuming, with significant tool cleaning, which presents a significant possibility of cross contamination. To address these issues, we developed an extension of the solvent-free method that replaces the mortar and pestle grinding with ball milling the sample in a glass vial with two small steel balls. This new method generates mass spectra with equal quality of the previous methods, but has significant advantages in productivity, eliminates cross contamination, and is applicable to liquid and soft or waxy analytes. (J Am Soc Mass Spectrom 2005, 16, 90-93) (c) 2004 American Society for Mass Spectrometry
\end{abstract}

$\mathrm{M}$ atrix-assisted laser desorption/ionization (MALDI) mass spectrometry [1-4] has become an important technique to characterize the chemical structure of many different analytes, including industrial polymer materials [5-10]. MALDI can generate important data on the telomer repeat units, end groups, and average molecular weights of these materials. MALDI methods have been developed to address a broad variety of polymer materials containing different chemistries. One of the key issues in traditional MALDI sample preparation is making good solutions of the analyte and the matrix [9]. Many interesting polymeric analytes are either completely insoluble or present significant challenges in making analytically useful solutions. To address these issues, solvent-free sample preparation methods have been developed [11-15]. Previous papers have demonstrated that solvent free sample preparations can produce analytically useful polymer MALDI data for insoluble analytes. Previous results of dry prepared polymer samples by Trimpin et al. show some advantages over traditional wet sample preparation methods [14]. They observed that the mass spectra obtained from this new sample preparation method had flatter baselines, higher signal-to-noise, and improved resolution.

Published online November 23, 2004

Address reprint requests to Dr. S. C. Hanton, Air Products and Chemicals Inc., 7201 Hamilton Blvd., Allentown, PA 18195, USA. E-mail: HantonSD@airproducts.com
Although the results of the previously published dry sample preparation methods produced excellent mass spectra, we found the mortar and pestle method to be very time-consuming, with significant tool cleaning, which presents a significant possibility of cross contamination. While a mini-ball mill can also address some of the labor issues, we have developed an extension of the dry method that replaces the mortar and pestle grinding with ball milling the sample in a small glass vial with two BBs (small steel balls). This new method generates mass spectra with equal quality of the previous methods, but has significant advantages in productivity, reduced cross contamination, and application to liquid and soft or waxy analytes. Our method also does not require the investment in a mini-ball mill.

\section{Experimental}

\section{Chemicals}

The polyethylene glycol (PEG) samples were obtained from Aldrich Chemical Inc. (Milwaukee, WI). The polymethylmethacrylate (PMMA) samples were obtained from American Polymer Standards (Mentor, OH). The polystyrene (PS) samples were obtained from American Polymer Standards and the National Institute of Standards and Technology (NIST, Gaithersburg, MD). The samples were prepared for MALDI using the following matrices: 2,5-dihydroxybenzoic acid (DHB), $\alpha$-cyano-4-hydroxycinnamic acid (CHCA), ferulic acid 
(FA), indole acrylic acid (IAA), and retinoic acid (Ret A), all of which were obtained from Aldrich. All of the matrices were of a standard grade. No high purity grades were used or additional purification done. Samples doped with a specific cationization agent used sodium chloride $(\mathrm{NaCl})$ or silver trifluoroacetate (AgTFA), both obtained from Aldrich. Tetrahydrofuran (THF) was obtained from Fisher Scientific (Fair Lawn, NJ). All chemicals were used as received.

\section{Sample Preparation: Wet}

For the traditional wet sample preparation using solvent, we made a $5 \mathrm{mg} / \mathrm{ml}$ solution of PS 5050 (average molecular weight is $5050 \mathrm{D})$, a $5 \mathrm{mg} / \mathrm{ml}$ solution of AgTFA, and a $0.25 \mathrm{M}$ solution of Ret A all in THF. We mixed these solutions by volume 1:1:7 and spotted $0.2 \mu \mathrm{l}$ on the target. The sample was allowed to air dry under ambient conditions.

\section{Sample Preparation: Mortar and Pestle}

For the dry preparation using a mortar and pestle, we ground together $5 \mathrm{mg}$ of PS 5050, $4 \mathrm{mg}$ of AgTFA, and $33 \mathrm{mg}$ of Ret A with a mortar and pestle for about $5 \mathrm{~min}$. We applied a few grains of the mixture to the target and pressed it to form a thin film with a small spatula.

\section{Sample Preparation: Novel Dry Method}

The new method involves adding the dry components of the sample (analyte, matrix, and cationization agent) to a small glass vial adding two small metal balls (commonly called BBs), capping the vial and mixing the contents on a vortex mixer for 30-60 s. Here is a more detailed standard procedure:

- Obtain a small glass vial (VL100, $15 \times 45$ mm, 1/8 oz, 1 dram, type 1 glass from All Pak, Bala Cynwyd, PA).

- Add 0.1-1.0 mg of analyte to the vial.

- Add an equal mass of cationization agent to the vial as analyte.

- Add 20-80 mg of matrix to the vial.

- Add two BBs (premium grade, $4.5 \mathrm{~mm}$ Zn plated, ultra smooth, steel air gun shot from Daisy Outdoor Products, Rogers, AR ) to the vial.

- Cap the vial.

- Hold the vial against the moving head of a vortex mixer (Vortex-Genie 2, model G-560 from Scientific Industries, Inc., Bohemia, NY) so that the two BBs rapidly move inside the vial. A combination of the variable speed of the mixer and how the vial is held against the mixer head will affect the BB motion. We have seen two primary motions of the BBs in the vial. They will either bounce mostly parallel with the sides, or they will race around the base of the vial. A combination of both motions seems to work best.

- Vortex for 30-60 s.
- Examine the base of the vial for unmixed material in the space between the square edge of the bottom of the vial and the race of the round BBs around the side of the vial.

- If necessary, use a small spatula to move material from the corner of the base of the vial up into the race defined by the BBs circuiting the vial. Then vortex the mixture again for 30-60 s.

- Use a small spatula to remove a few grains of the mixed sample.

- Tap the spatula on the metal MALDI target to transfer a couple of grains to the MALDI target.

- Use the flat edge of the spatula to spread the sample under moderate pressure until a thin film about 2-3 mm in diameter is created. Use a circular motion with enough pressure to slightly bend the blade of the micro-spatula.

- Firmly tap the MALDI target on edge against the table top to knock off most of the remaining mixture. To minimize cross contamination of samples on the target, load the target from the bottom up.

- Analyze the target by MALDI.

\section{Mass Spectrometry}

All of the MALDI experiments were conducted on a Bruker Biflex III (Billerica, MA) TOF mass spectrometer. These experiments were all conducted in reflectron mode using delayed extraction. The Biflex was equipped with a nitrogen laser operated at $337 \mathrm{~nm}$ and $3 \mathrm{~Hz}$. The laser fluence was optimized to be slightly above threshold for ions. The laser fluence was optimized differently for each matrix molecule used depending on their relative absorptivities. The source conditions of the mass spectrometer were optimized for mass resolution near the peak of the polymer telomer distribution. Spectra were collected by moving the laser around the surface of the sample and averaging 125 shots/spectrum. The data were analyzed using Polymerix software (Sierra Analytics, Modesto, CA).

\section{Results and Discussion}

In their recent paper, Trimpin et al. show that using solvent-free sample preparation methods for MALDI samples improved mass spectra can be produced [14]. We have reproduced their results as demonstrated in Figure 1. Figure 1 shows MALDI mass spectra produced for a sample of PS 5050 using the mortar and pestle solvent-free preparation method (top) and a typical solvent-based sample preparation (bottom). In both cases the sample was prepared with Ret A as the matrix and AgTFA as the cationization agent. In Figure 1 we can clearly see the improvements in the mass spectra, as reported by Trimpin, et al. We observe a flatter baseline, improved signal-to-noise, and improved mass resolution.

In our subsequent use of the mortar and pestle solvent-free sample preparation method to analyze in- 


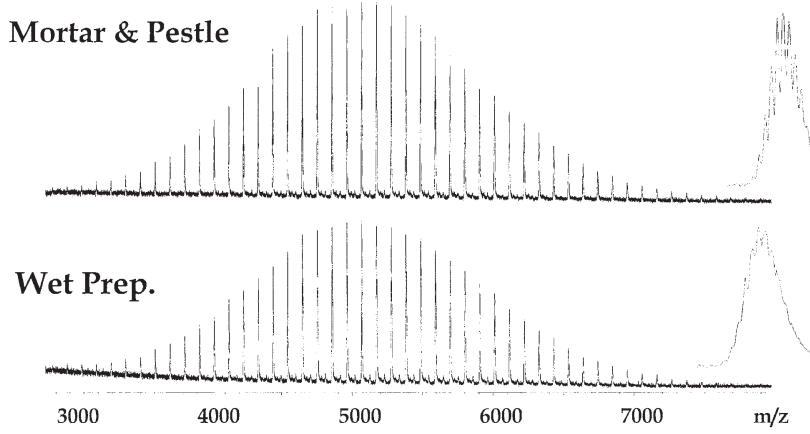

Figure 1. MALDI mass spectra produced for a sample of PS 5050 using the mortar and pestle dry preparation method (top) and a typical solvent-based sample preparation (bottom). In both cases, the sample was prepared with Ret A as the matrix and AgTFA as the cationization agent. The dry prepared spectrum shows higher signal intensity, improved signal-to-noise, and a flatter baseline. The expanded spectra are from the oligomers at the peaks of the distributions.

soluble polymer samples, we found several problems with the method:

\section{- Inefficiency}

The grinding took many minutes.

We only owned a few mortar and pestle sets (cost is a factor).

It was inefficient to transfer the mixed sample to a permanent storage vessel.

It was difficult and time consuming to clean the mortar and pestle.

We could only analyze a few samples per day with this method.

- High chance of cross contamination

Because the analytes were insoluble and the MALDI technique is extremely sensitive, it was very difficult to completely remove each analyte from the mortar and pestle.

- Could only analyze "hard" analytes.

Only analytes that were sufficiently brittle would grind effectively in the mortar and pestle [16].

To address these problems, we have developed a new extension of the previously published solvent-free sample preparation methods for MALDI. Our new method addresses all of the above concerns. It is relatively rapid, uses inexpensive and disposable tools, and we have demonstrated its utility on a variety of analytes including liquids and waxes. The new method was described in detail above in the experimental section.

In this method, the BBs behave as a mini-ball mill to effectively grind together the analyte, matrix, and cationization agent. The method is fast and effective.

Figure 2 shows MALDI mass spectra of the PS 5050 sample prepared by both the mortar and pestle method (top) and our new BB method (bottom). In both cases, the sample was prepared with DHB as the matrix and AgTFA as the cationization agent. The spectra are analytically identical. Using the new BB method, we

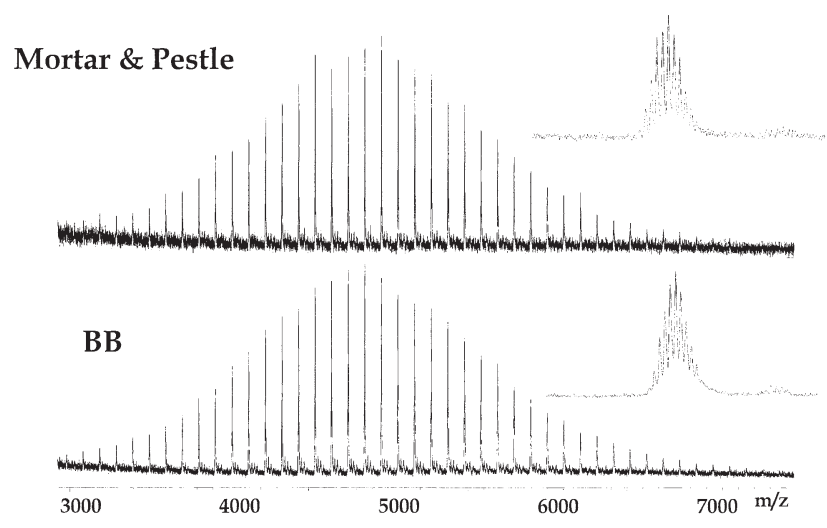

Figure 2. MALDI mass spectra produced for a sample of PS 5050 using the mortar and pestle dry preparation method (top) and the new BB solvent-free sample preparation (bottom). In both cases, the sample was prepared with DHB as the matrix and AgTFA as the cationization agent. The two spectra are nearly identical. The expanded spectra are from the oligomers at the peaks of the distributions.

have successfully generated high quality PS 5050 MALDI data from a variety of different matrices (results not shown). This example, prepared with DHB, provides the best mass resolution. We have retained the benefits in the mass spectrum obtained by solvent-free sample preparation, but greatly increased the speed and ease of doing the experiment.

We can also apply this method to a wide variety of different analytes. In Figure 3 we show mass spectra for various PEG samples prepared using the BB method. The lowest mass sample, PEG 620, is a liquid and the other samples range from waxy to brittle solids. Each sample produces an excellent MALDI mass spectrum. We are no longer constrained to either "hard" analytes or forced to use cryogenic grinding to prepare samples.

In our laboratory, we have applied this new method to many other insoluble samples including solvent-free polymers, dispersions of insoluble polymers in water,

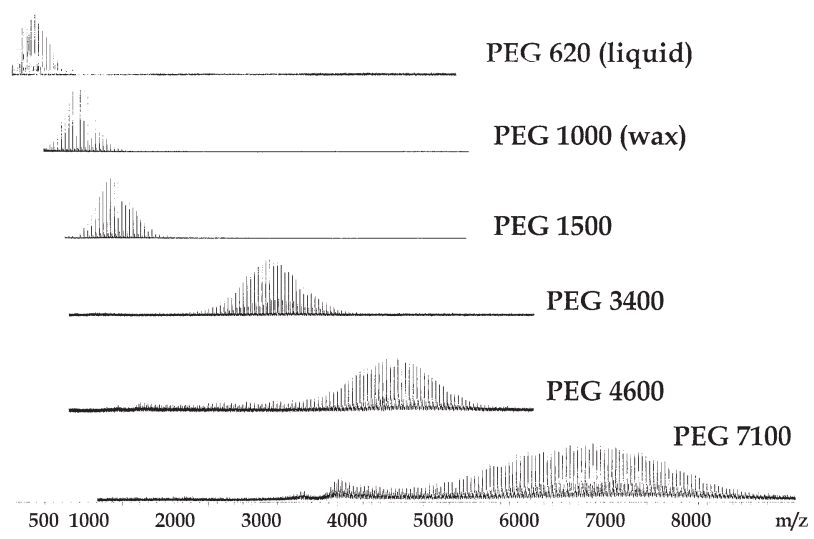

Figure 3. MALDI mass spectra for various PEG samples (PEG 620-PEG 7100) prepared using the BB method. The lowest mass sample, PEG 620, is a liquid and the other samples are waxy solids. Each sample produces an excellent MALDI mass spectrum with the BB method. 


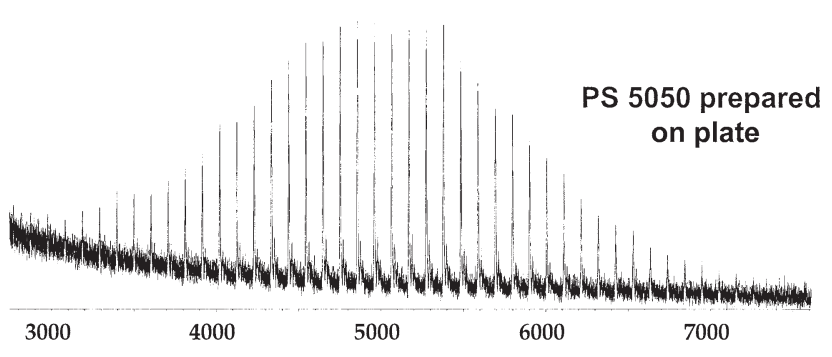

Figure 4. MALDI mass spectrum for PS 5050 ground on the metal MALDI target with Ret A and AgTFA using significantly less than $0.1 \mathrm{mg}$ of PS 5050 .

and even smudges of polymer samples stuck to pieces of tissue paper. For the dispersion samples, we measure the weight percent solids in the dispersion and then calculate the mass of dispersion required to add $0.1 \mathrm{mg}$ of analyte to the sample. For a $1 \%$ solids dispersion this is $10 \mu \mathrm{l}$. We add the $10 \mu \mathrm{l}$ of dispersion to the vial and prepare as if it were solvent-free. The water does not appear to interfere with the MALDI results. For the sample stuck to the tissue paper, we carefully cut out the areas with the smudges, cut them into small pieces and added them to the vial. While the tissue paper did not grind effectively on the vortex mixer, we did successfully obtain a spectrum from the polymer sample. This spectrum matched a spectrum obtained from a standard produced later.

As we continue to improve our methods and look for even faster sample preparation methods that require even less analyte, we have had initial success grinding an analyte and matrix directly on the MALDI target. Figure 4 shows a MALDI mass spectrum for PS 5050 ground on the metal MALDI target with Ret A and AgTFA. Significantly less than $0.1 \mathrm{mg}$ of analyte was used in this preparation and we did obtain an analytically useful mass spectrum from this experiment. Perhaps future innovations will bring the mass spectral quality up to the BB method quality.

\section{Acknowledgments}

The authors thank Air Products and Chemicals, Inc. for their support of this research, Dr. Lorenz Brunner of NIST for teaching the authors their first solvent-free sample preparation, Professor Kevin Owens and his group from Drexel University for many helpful discussions about MALDI sample preparation, Dr. Sarah Trimpin from Oregon State University for helpful discussions of her solvent-free sample preparation technique, Dr. Seksan Dheandanoo for acquiring some of the data presented here, and Dr. John Sadowski for critical review of the manuscript.

\section{References}

1. Tanaka, K.; Waki, H.; Ido, Y.; Akita, S.; Yoshido, Y.; Yoshido, T. Protein and Polymer Analyses up to $m / z$ 100,000 by Laser ionization time-of-flight mass spectrometry. Rapid Commun. Mass Spectrom. 1988, 2, 151.

2. Karas, M.; Hillenkamp, F. Laser desorption ionization of proteins with molecular masses exceeding 10,000 daltons. Anal. Chem. 1988, 60, 2299.

3. Bahr, U.; Deppe, A.; Karas, M.; Hillenkamp, F.; Giessman, U. Mass spectrometry of synthetic polymers by UV-matrix-assisted laser desorption/ionization. Anal. Chem. 1992, 64, 2866.

4. Danis, P.; Karr, D.; Mayer, F.; Holle, A.; Watson, C. The analysis of water-soluble polymers by matrix-assisted laser desorption time-of-flight mass spectrometry. Org. Mass Spectrom. 1992, 27, 843.

5. Hanton, S. D. Mass spectrometry of polymers and polymer surfaces. Chem. Rev. 2001, 101(2), 527.

6. Nielen, M. W. F. MALDI time-of-flight mass spectrometry of synthetic polymers. Mass Spectrom. Rev. 1999, 18, 309.

7. Montaudo, G.; Lattimer, R. P., Eds.; Mass Spectrometry of Polymers; CRC Press: Boca Raton, FL, 2002.

8. Pash, H.; Schrepp, W., Eds.; MALDI-TOF Mass Spectrometry of Synthetic Polymers; Springer-Verlag: Berlin, 2003.

9. Wallace, W. E.; Guttman, C. M.; Hanton, S. D. Quantitative Synthetic Polymer Mass Spectrometry Workshop. J. Res. Natl. Inst. Stand. Technol. 2003, 108, 79-85.

10. Wallace, W. E.; Guttman, C. M.; Wetzel, S. J.; Hanton, S. D. Mass Spectrometry of Synthetic-Polymer Mixtures Workshop. Rapid Commun. Mass Spectrom. 2004, 18, 518-521.

11. Skelton, R.; Dubois, F.; Zenobi, R. A MALDI sample preparation method suitable for insoluble polymers. Anal. Chem. 2000, 72, 1707-1710.

12. Przybilla, L.; Brand, J.-D.; Yoshimura, K.; Räder, H. J.; Müllen, K. MALDI-TOF mass spectrometry of insoluble giant polycyclic aromatic hydrocarbons by a new method of sample preparation. Anal. Chem. 2000, 72, 4591-4597.

13. Marie, A.; Fournier, F.; Tabet, J. C. Characterization of synthetic polymers by MALDI-TOF/MS: Investigation into new methods of sample target preparation and consequence on mass spectrum finger print. Anal. Chem. 2000, 72, 5106-5114.

14. Trimpin, S.; Rouhanipour, A.; Räder, H. J.; Müllen, K. New aspects in matrix-assisted laser desorption/ionization time-offlight mass spectrometry: A universal solvent-free sample preparation. Rapid Commun. Mass Spectrom. 2001, 15, 13641373.

15. Gies, A. P.; Nonidez, W. K.; Anthamatten, M.; Cook, R. C.; Mays, J. W. Characterization of an insoluble polyimide oligomer by matrix-assisted laser desorption/ionization time-offlight mass spectrometry. Rapid Commun. Mass Spectrom. 2002, 16, 1903-1910.

16. Personal communication with Dr. Lorenz Brunner while he was working at the National Institute of Standards and Technology (NIST). Dr. Brunner taught us the mortar and pestle method and coached us on its applications. 